\title{
ALIENATION OF THE JENSEN, CAUCHY AND D'ALEMBERT EQUATIONS
}

\author{
BARBARA SOBEK
}

\begin{abstract}
Let $(S,+)$ be a commutative semigroup, $\sigma: S \rightarrow S$ be an endomorphism with $\sigma^{2}=i d$ and let $K$ be a field of characteristic different from 2 . Inspired by the problem of strong alienation of the Jensen equation and the exponential Cauchy equation, we study the solutions $f, g: S \rightarrow K$ of the functional equation

$$
f(x+y)+f(x+\sigma(y))+g(x+y)=2 f(x)+g(x) g(y) \quad \text { for } \quad x, y \in S .
$$

We also consider an analogous problem for the Jensen and the d'Alembert equations as well as for the d'Alembert and the exponential Cauchy equations.
\end{abstract}

\section{Introduction}

The notions of alienation and strong alienation, introduced by J. Dhombres [1], describe the phenomenon that a functional equation, resulting from adding up two functional equations side by side, splits back into the system of these two equations. More precisely, let $E_{1}(f)=0$ and $E_{2}(f)=0$ be two functional equations for a function $f: X \rightarrow Y$, where $X$ is a nonempty set and $(Y,+, 0)$ is a monoid. Following J. Dhombres [1] and Gy. Maksa and M. Sablik [8, we say that the equations $E_{1}$ and $E_{2}$ are alien with respect to $X$ and $Y$, if every

Received: 1.04.2016. Revised: 20.05.2016. Accepted: 21.05.2016.

(2010) Mathematics Subject Classification: 39B52, 39B72.

Key words and phrases: Alienation, exponential Cauchy equation, Jensen equation, d'Alembert equation. 
solution $f: X \rightarrow Y$ of equation

$$
E_{1}(f)+E_{2}(f)=0
$$

solves the system

$$
\left\{\begin{array}{l}
E_{1}(f)=0 \\
E_{2}(f)=0 .
\end{array}\right.
$$

The equations $E_{1}$ and $E_{2}$ are strongly alien whenever a pair $(f, g)$ is a solution of

$$
E_{1}(f)+E_{2}(g)=0
$$

only if the system

$$
\left\{\begin{array}{l}
E_{1}(f)=0 \\
E_{2}(g)=0
\end{array}\right.
$$

holds.

The problems of alienation and strong alienation of functional equations have been studied by many authors (see e.g. [1]-8]). In particular, as regards the additive and exponential Cauchy equations, R. Ger [3] showed, in a quite general setting, that a pair of functions $(f, g)$, satisfying functional equation

$$
f(x+y)+g(x+y)=f(x)+f(y)+g(x) g(y),
$$

need not solve the system

$$
\left\{\begin{array}{l}
f(x+y)=f(x)+f(y) \\
g(x+y)=g(x) g(y)
\end{array}\right.
$$

unless $f$ and $g$ satisfy some additional assumption. Thus, in general, the additive and exponential Cauchy equations are not strongly alien in the sense of Dhombres. A similar problem for the exponential and logarithmic Cauchy equations has been considered by Z. Kominek and J. Sikorska [7]. In a recent paper [8] Gy. Maksa and M. Sablik provided a sufficient condition for the strong alienation of the exponential Cauchy equation and the Hosszú equation.

Inspired by these results, we investigate the problem of strong alienation for further pairs of classical functional equations. More precisely, assuming that $K$ is a field of characteristic different from $2,(S,+)$ is a commutative 
semigroup and $\sigma$ is an endomorphism of $S$ with $\sigma(\sigma(x))=x$ for $x \in S$, in the next section we study the solutions $f, g: S \rightarrow K$ of the functional equation

$$
f(x+y)+f(x+\sigma(y))+g(x+y)=2 f(x)+g(x) g(y) \quad \text { for } x, y \in S
$$

resulting from summing up the Jensen equation

$$
f(x+y)+f(x+\sigma(y))=2 f(x) \quad \text { for } x, y \in S
$$

and the Cauchy equation

$$
g(x+y)=g(x) g(y) \quad \text { for } x, y \in S
$$

side by side. We show that (1.1) forces $f$ and $g$ to solve the system (1.2)-(1.3), which means that equations (1.2) and (1.3) are strongly alien. In the third section we prove that the phenomenon of strong alienation holds also for the pair consisting of equation 1.2 and the following generalized version of the classical d'Alembert equation (cf. [10])

$$
h(x+y)+h(x+\sigma(y))=2 h(x) h(y) \quad \text { for } x, y \in S .
$$

In the last section we study the problem of strong alienation of equations (1.3) and (1.4). It turns out that, contrary to the previous pairs, these equations, in general, are not strongly alien. We present a sufficient condition for the strong alienation of (1.3) and (1.4).

Throughout the paper, unless otherwise stated, $(S,+)$ is a commutative semigroup, $\sigma$ is an endomorphism of $S$ with $\sigma(\sigma(x))=x$ for $x \in S$ and $K$ is a field of characteristic different from 2. In what follows, we will write $\sigma x$ instead of $\sigma(x)$.

\section{Jensen and Cauchy equations}

The following result states that the Jensen equation and the exponential Cauchy equation are strongly alien in the sense of Dhombres.

THEOREM 2.1. Assume that a pair of functions $(f, g)$, where $f, g: S \rightarrow K$, satisfies equation (1.1). Then $f$ solves 1.2 and $g$ satisfies (1.3). 
Proof. If $g=0$ or $g=1$, then $g$ satisfies equation (1.3), which implies that $f$ solves 1.2 . So, assume that $g \neq 0$ and $g \neq 1$. In the proof we apply some ideas from [9]. Making use of (1.1), for every $x, y, z \in S$, we get

$$
\begin{gathered}
(f+g)(x+y+z)+f(x+y+\sigma z)=2 f(x+y)+g(x+y) g(z) \\
(f+g)(x+\sigma y+z)+f(x+\sigma y+\sigma z)=2 f(x+\sigma y)+g(x+\sigma y) g(z) \\
(f+g)(x+y+z)+f(x+\sigma y+\sigma z)=2 f(x)+g(x) g(y+z)
\end{gathered}
$$

and

$$
(f+g)(x+\sigma y+z)+f(x+y+\sigma z)=2 f(x)+g(x) g(\sigma y+z) .
$$

Summing up equalities (2.1) and (2.2) side by side, and subtracting from the equality thus obtained the sum of equalities (2.3) and (2.4), we infer that

$$
\begin{aligned}
& 2[f(x+y)+f(x+\sigma y)]+[g(x+y)+g(x+\sigma y)] g(z) \\
& \quad=4 f(x)+g(x)[g(z+y)+g(z+\sigma y)] \quad \text { for } x, y, z \in S .
\end{aligned}
$$

Thus, applying (1.1) again, we obtain

$$
\begin{aligned}
& {[g(x+y)+g(x+\sigma y)] g(z)-2 g(x+y)} \\
& \quad=g(x)[g(z+y)+g(z+\sigma y)-2 g(y)] \quad \text { for } \quad x, y, z \in S
\end{aligned}
$$

Replacing in 2.5 y by $\sigma y$, we get

$$
\begin{aligned}
{[g(x+\sigma y)+} & g(x+y)] g(z)-2 g(x+\sigma y) \\
& =g(x)[g(z+\sigma y)+g(z+y)-2 g(\sigma y)] \quad \text { for } \quad x, y, z \in S
\end{aligned}
$$

Hence, subtracting (2.5) from (2.6) side by side, we arrive at

$$
g(x+y)-g(x+\sigma y)=g(x)[g(y)-g(\sigma y)] \quad \text { for } x, y \in S,
$$

whereas by summing up (2.5) and (2.6) side by side, we get

$$
\begin{aligned}
& {[g(x+y)+g(x+\sigma y)] \cdot[g(z)-1]} \\
& =g(x)[g(z+y)+g(z+\sigma y)-g(y)-g(\sigma y)] \quad \text { for } x, y, z \in S
\end{aligned}
$$


Fix $x_{0}, z_{0} \in S$ with $g\left(x_{0}\right) \neq 0$ and $g\left(z_{0}\right) \neq 1$ and define a function $U: S \rightarrow K$ in the following way

$$
U(y)=\frac{g\left(x_{0}+y\right)+g\left(x_{0}+\sigma y\right)}{g\left(x_{0}\right)} \quad \text { for } y \in S .
$$

Then

$$
U(\sigma y)=U(y) \text { for } y \in S
$$

and, by (2.8),

(2.10) $g(x+y)+g(x+\sigma y)-g(y)-g(\sigma y)=U(y)[g(x)-1]$ for $x, y \in S$.

Furthermore, in view of (2.8), we have

$$
U(y)=\frac{g\left(z_{0}+y\right)+g\left(z_{0}+\sigma y\right)-g(y)-g(\sigma y)}{g\left(z_{0}\right)-1} \quad \text { for } y \in S
$$

and

$$
g(x+y)+g(x+\sigma y)=g(x) U(y) \quad \text { for } x, y \in S .
$$

So, from 2.7 and 2.11 it follows that

$$
2 g(x+y)=g(x) V(y) \quad \text { for } x, y \in S,
$$

where the function $V: S \rightarrow K$ is defined by

$$
V(y)=U(y)+g(y)-g(\sigma y) \quad \text { for } y \in S .
$$

Since, in view of 2.9$), V(y)+V(\sigma y)=2 U(y)$ for $y \in S$, making use of 2.10) and (2.12), we obtain

$$
\begin{aligned}
2 g(x) U(y) & =g(x) V(y)+g(x) V(\sigma y)=2 g(x+y)+2 g(x+\sigma y) \\
& =2 U(y)[g(x)-1]+2 g(y)+2 g(\sigma y) \quad \text { for } x, y \in S
\end{aligned}
$$

Hence $U(y)=g(y)+g(\sigma y)$ for $y \in S$, which together with $(2.13)$ gives $V=2 g$. Thus, taking into account (2.12), we conclude that $g$ satisfies $(1.3)$ and so, in view of (1.1), $f$ solves 1.2 .

From Theorem 2.1 and [10, Theorem 2] we derive the following result. 
Corollary 2.2. Assume that a pair $(f, g)$ of functions mapping $S$ into $K$ satisfies equation (1.1). Then $g$ satisfies equation (1.3) and there exist a constant $c \in K$ and an additive function $a: S \rightarrow K$ such that $a(\sigma x)=-a(x)$ for $x \in S$ and $f(x)=a(x)+c$ for $x \in S$.

\section{Jensen and d'Alembert equations}

The following result shows that the phenomenon of strong alienation takes place also in the case of the Jensen and the d'Alembert equations.

THEOREM 3.1. Assume that a pair of functions $(f, h)$, where $f, h: S \rightarrow K$, satisfies equation

$$
(f+h)(x+y)+(f+h)(x+\sigma y)=2 f(x)+2 h(x) h(y) \quad \text { for } x, y \in S .
$$

Then $f$ satisfies (1.2) and $h$ solves (1.4).

Proof. If $h=0$ or $h=1$ then $h$ satisfies $(1.4)$, which forces $f$ to satisfy (1.2). So, assume that $h \neq 0$ and $h \neq 1$. In view of 3.1 , for every $x, y, z \in S$, we have

$$
\begin{gathered}
(f+h)(x+y+z)+(f+h)(x+y+\sigma z)=2 f(x+y)+2 h(x+y) h(z), \\
(f+h)(x+\sigma y+z)+(f+h)(x+\sigma y+\sigma z)=2 f(x+\sigma y)+2 h(x+\sigma y) h(z), \\
(f+h)(x+y+z)+(f+h)(x+\sigma y+\sigma z)=2 f(x)+2 h(x) h(y+z)
\end{gathered}
$$

and

$$
(f+h)(x+y+\sigma z)+(f+h)(x+\sigma y+z)=2 f(x)+2 h(x) h(y+\sigma z) .
$$

Furthermore, arguing as in the proof of Theorem 2.1, from these equalities we derive

$$
\begin{aligned}
2[f(x+y)+f(x+\sigma y)]+2 h(z)[h(x+y)+h(x+\sigma y)] & \\
& =4 f(x)+2 h(x)[h(y+z)+h(y+\sigma z)] \quad \text { for } x, y, z \in S .
\end{aligned}
$$


Thus, applying (3.1) again, we get

$$
\begin{aligned}
2[h(z)-1] \cdot[ & h(x+y)+h(x+\sigma y)] \\
& =2 h(x)[h(y+z)+h(y+\sigma z)-2 h(y)] \text { for } x, y, z \in S
\end{aligned}
$$

Let $x_{0}, z_{0} \in S$ be such that $h\left(x_{0}\right) \neq 0$ and $h\left(z_{0}\right) \neq 1$. Define a function $\varphi: S \rightarrow K$ by

$$
\varphi(y)=\frac{h\left(x_{0}+y\right)+h\left(x_{0}+\sigma y\right)}{2 h\left(x_{0}\right)} \quad \text { for } y \in S
$$

Then 3.2 implies that

$$
\varphi(y)=\frac{h\left(y+z_{0}\right)+h\left(y+\sigma z_{0}\right)-2 h(y)}{2\left[h\left(z_{0}\right)-1\right]} \text { for } y \in S .
$$

Thus, setting in $3.2 z=z_{0}$ and $x=x_{0}$, we obtain

$$
h(x+y)+h(x+\sigma y)=2 h(x) \varphi(y) \quad \text { for } x, y \in S
$$

and

$$
h(y+z)+h(y+\sigma z)=2 h(y)+2[h(z)-1] \varphi(y) \text { for } y, z \in S
$$

respectively. From the last two equalities it follows that

$$
h(x)[\varphi(y)-1]=[h(y)-1] \varphi(x) \text { for } x, y \in S
$$

So, letting $\lambda=\frac{\varphi\left(z_{0}\right)-1}{h\left(z_{0}\right)-1}$, we get $\varphi=\lambda h$. Hence, making use of (3.4), we obtain $(\lambda-1) h(x)=0$ for $x \in S$. Thus, as $h \neq 0$, we have $\lambda=1$, which means that $\varphi=h$. Therefore, from 3.3 we derive that $h$ satisfies equation (1.4) and so, in view of (3.1), $f$ satisfies $(1.2)$.

Applying [10, Theorems 1-2], from Theorem 3.1 we deduce the following result.

COROLlary 3.2. Let $K$ be a quadratically closed field of characteristic different from 2. Suppose that a pair of functions $(f, h)$, where $f, h: S \rightarrow$ $K$, satisfies equation (3.1). Then there exist a function $g: S \rightarrow K$ satisfying 
equation 1.3, an additive function $a: S \rightarrow K$ and a constant $c \in K$ such that $a(\sigma x)=-a(x)$ for $x \in S, f(x)=a(x)+c$ for $x \in S$ and

$$
h(x)=\frac{g(x)+g(\sigma x)}{2} \quad \text { for } x \in S .
$$

\section{Cauchy and d'Alembert equations}

In this section we study the alienation problem for the pair of equations (1.3) and (1.4). The following example shows that, in general, these equations are not strongly alien to each other.

ExAmple. Let $g, h: S \rightarrow \mathbb{C}$ be the constant functions, say $g=c$ and $h=d$, where $c, d \in \mathbb{C} \backslash\{0,1\}$ are such that $c(1-c)=2 d(d-1)$. Then, as one can easily check, the pair $(g, h)$ satisfies the equation resulting from adding up (1.3) and (1.4) side by side, but neither $g$ fulfills $(1.3)$, nor $h$ satisfies (1.4).

Now, we are going to show that under some additional assumptions, equations $(1.3)$ and $(1.4)$ are strongly alien to each other. To this end, we will need the following simple result.

Lemma 4.1. Let $(S,+, 0)$ be a commutative monoid. Assume that a pair of functions $(g, h)$, where $g, h: S \rightarrow K$, satisfies equation

$$
g(x+y)+h(x+y)+h(x+\sigma y)=g(x) g(y)+2 h(x) h(y) \quad \text { for } x, y \in S
$$

Then $g$ satisfies 2.7) and $h$ is even with respect to $\sigma$, i.e.

$$
h(x)=h(\sigma x) \quad \text { for } x \in S .
$$

Proof. Since the monoid $(S,+, 0)$ is commutative, from (4.1) we derive that

$$
h(x+\sigma y)=h(y+\sigma x) \text { for } x, y \in S .
$$

Applying this equality with $y=0$, we obtain 4.2 . Furthermore, replacing in (4.1) $y$ by $\sigma y$ and making use of 4.2, we get

(4.3) $g(x+\sigma y)+h(x+\sigma y)+h(x+y)=g(x) g(\sigma y)+2 h(x) h(y) \quad$ for $x, y \in S$.

Subtracting (4.3) from 4.1 side by side, we infer that $g$ satisfies (2.7). 
THEOREM 4.2. Let $(S,+, 0)$ be a commutative monoid. Assume that a pair of functions $(g, h)$, where $g, h: S \rightarrow K$, satisfies equation (4.1) and $g\left(s_{0}\right) \neq$ $g\left(\sigma s_{0}\right)$ for some $s_{0} \in S$. Then $g$ satisfies (1.3) and h satisfies (1.4).

Proof. Let a function $g^{o}: S \rightarrow K$ be given by $g^{o}(x)=g(x)-g(\sigma x)$ for $x \in S$. Note that $g^{o}\left(s_{0}\right) \neq 0$. Moreover, applying Lemma 4.1, we obtain

$$
g(x+y)-g(x+\sigma y)=g(x) g^{o}(y) \quad \text { for } x, y \in S .
$$

Setting in (4.4) $x=0$, we get $g^{o}(y)=g(0) g^{o}(y)$ for $y \in S$. Since $g^{o}\left(s_{0}\right) \neq 0$, this yields $g(0)=1$. Furthermore, using (4.1), for every $x, y, z \in S$, we have

$$
\begin{gathered}
(g+h)(x+y+z)+h(x+y+\sigma z)=g(x+y) g(z)+2 h(x+y) h(z), \\
(g+h)(x+\sigma y+z)+h(x+\sigma y+\sigma z)=g(x+\sigma y) g(z)+2 h(x+\sigma y) h(z), \\
(g+h)(x+y+z)+h(x+\sigma y+\sigma z)=g(x) g(y+z)+2 h(x) h(y+z)
\end{gathered}
$$

and

$$
(g+h)(x+\sigma y+z)+h(x+y+\sigma z)=g(x) g(\sigma y+z)+2 h(x) h(\sigma y+z) .
$$

Hence, arguing as in the proof of Theorem 2.1, from the above equalities we obtain

$$
\begin{aligned}
& {[g(x+y)+g(x+\sigma y)] g(z)+2[h(x+y)+h(x+\sigma y)] h(z)} \\
& =g(x)[g(z+y)+g(z+\sigma y)]+2 h(x)[h(z+y)+h(z+\sigma y)] \text { for } x, y, z \in S .
\end{aligned}
$$

According to Lemma 4.1, $h$ satisfies 4.2. Therefore, replacing in the last equality $z$ by $\sigma z$ and subtracting the equality thus obtained from the original one, we get

$$
[g(x+y)+g(x+\sigma y)] g^{o}(z)=g(x)\left[g^{o}(z+y)+g^{o}(z+\sigma y)\right] \quad \text { for } x, y, z \in S .
$$

Let a function $U: S \rightarrow K$ be defined in the following way

$$
U(y)=\frac{g^{o}\left(s_{0}+y\right)+g^{o}\left(s_{0}+\sigma y\right)}{g^{o}\left(s_{0}\right)} \quad \text { for } y \in S .
$$

Then, in view of (4.5), we have

$$
g(x+y)+g(x+\sigma y)=g(x) U(y) \quad \text { for } x, y \in S .
$$


Consequently, taking into account 4.4, we obtain

$$
2 g(x+y)=g(x)\left[g^{o}(y)+U(y)\right] \quad \text { for } x, y \in S
$$

Furthermore, as $g(0)=1$, setting in 4.6 $x=0$, we conclude that $g(y)+$ $g(\sigma y)=U(y)$ for $y \in S$. Thus $g^{o}(y)+U(y)=2 g(y)$ for $y \in S$ and so, in view of 4.7), $g$ satisfies equation (1.3). Hence, from (4.1) it follows that $h$ satisfies (1.4).

We conclude the paper with a result which states that in the class of nonconstant functions mapping a 2-divisible Abelian group into a field of characteristic different from 2, the exponential Cauchy equation and the d'Alembert equation are strongly alien to each other.

Corollary 4.3. Let $(S,+)$ be an Abelian group with $S=2 S$. Assume that a pair of functions $(g, h)$, where $g, h: S \rightarrow K$, satisfies equation

$$
g(x+y)+h(x+y)+h(x-y)=g(x) g(y)+2 h(x) h(y) \quad \text { for } x, y \in S
$$

Then one of the following two alternatives holds:

(i) there exist $c, d \in K$ with $c(1-c)=2 d(d-1)$ such that $g=c$ and $h=d$;

(ii) $g$ satisfies (1.3) and $h$ satisfies equation

$$
h(x+y)+h(x-y)=2 h(x) h(y) \quad \text { for } x, y \in S .
$$

Proof. First, consider the case where $g$ is nonconstant. Suppose that $g(s)=g(-s)$ for $s \in S$. Then, applying Lemma 4.1 we obtain that $g(x+y)=$ $g(x-y)$ for $x, y \in S$. Thus $g(2 x)=g(0)$ for $x \in S$. Since $2 S=S$, this means that $g=g(0)$, which yields a contradiction. Therefore, there is an $s_{0} \in S$ with $g\left(s_{0}\right) \neq g\left(-s_{0}\right)$. So, applying Theorem 4.2 , we obtain (ii).

Now, assume that $g$ is constant, say $g=c$ for some $c \in K$. If $c \in\{0,1\}$ then $g$ satisfies 1.3 , which implies that $h$ solves 4.9 . Consequently, (ii) holds. So, suppose that $c \notin\{0,1\}$. Then, letting $\alpha:=c^{2}-c$, we have $\alpha \neq 0$. Furthermore, taking into account (4.8), we obtain

$$
h(x+y)+h(x-y)=2 h(x) h(y)+\alpha \quad \text { for } x, y \in S .
$$

Thus, for every $x, y, z \in S$, we have

$$
\begin{aligned}
& h(x+y+z)+h(x-y-z)+h(x+y-z)+h(x-y+z) \\
& \quad=2 h(x) h(y+z)+2 h(x) h(y-z)+2 \alpha=4 h(x) h(y) h(z)+2 \alpha h(x)+2 \alpha .
\end{aligned}
$$


Making use of 4.10 again, for every $x, y, z \in S$, we obtain also

$$
\begin{aligned}
& h(x+y+z)+h(x+y-z)+h(x-y+z)+h(x-y-z) \\
& \quad=2 h(x+y) h(z)+2 h(x-y) h(z)+2 \alpha=4 h(x) h(y) h(z)+2 \alpha h(z)+2 \alpha .
\end{aligned}
$$

From these equalities we derive that $\alpha h(x)=\alpha h(z)$ for $x, z \in S$. Since $\alpha \neq 0$, this means that $h$ is constant, say $h=d$. Therefore, taking into account (4.8), we get $c(1-c)=2 d(d-1)$, and so (ii) is valid.

\section{References}

[1] Dhombres J., Relations de dépendance entre les équations fonctionnelles de Cauchy, Aequationes Math. 35 (1988), 186-212.

[2] Fechner W., A characterization of quadratic-multiplicative mappings, Monatsh. Math. 164 (2011), 383-392.

[3] Ger R., Additivity and exponentiality are alien to each other, Aequationes Math. 80 (2010), 111-118.

[4] Ger R., The alienation phenomenon and associative rational operations, Ann. Math. Sil. 27 (2013), 75-88.

[5] Ger R., Alienation of additive and logarithmic equations, Ann. Univ. Sci. Budapest. Sect. Comput. 40 (2013), 269-274.

[6] Ger R., Reich L., A generalized ring homomorphisms equation, Monatsh. Math. 159 (2000), 225-233.

[7] Kominek Z., Sikorska J., Alienation of the logarithmic and exponential functional equations, Aequationes Math. 90 (2016), 107-121.

[8] Maksa Gy., Sablik M., On the alienation of the exponential Cauchy equation and the Hosszú equation, Aequationes Math. 90 (2016), 57-66.

[9] Sinopoulos P., Generalized sine equations I, Aequationes Math. 48 (1994), 171-193.

[10] Sinopoulos P., Functional equations on semigroups, Aequationes Math. 59 (2000), 255-261.

Faculty of Mathematics and Natural Sciences

University of Rzeszów

Pigonia 1

35-310 RzEszów

POLAND

e-mail: bsobek@ur.edu.pl 